Int. J. Contemp. Math. Sciences, Vol. 2, 2007, no. 22, 1069 - 1075

\title{
A Generalization of Lifting Modules
}

\author{
Y. Talebi \\ Department of Mathematics, \\ University of Mazandaran, \\ Babolsar, Iran, 47416-1468 \\ M. J. Nematollahi and Kh. Ghaziani \\ Islamic Azad University-Arsanjan Branch, \\ Arsanjan, Iran, 73761-168
}

\begin{abstract}
A module $M$ is called lifting if every submodule $N$ of $M$, contains a direct summand $K$ of $M$ such that $N / K \ll M / K$. We call a module $M$ cf-lifting if every coessentialy a finitely generated submodule of $M$ lies above a direct summand. In this paper we study about cf-lifting modules and give some conditions for a cf-lifting module to be a lifting module. At the end of this paper we obtain some properties of decomposition of cf-lifting modules.
\end{abstract}

Mathematics Subject Classification: 16D40, 16D90.

Keywords: lifting module, cf-lifting module, hollow-lifting module, local direct summand, relative projective.

\section{Introduction}

Extending modules are generalization of injective modules and, dually, lifting modules generalize projective supplemented modules. In this paper we generalize the lifting modules as cf-lifting modules.

\section{Preliminary Notes}

Throughout this paper, $R$ is always an associative ring with unit and $\operatorname{Mod}-R$ denotes the category of unital right R-modules. Let $M$ be a module and $S \leq M . S$ is called small in $M$ ( notation $S \ll M$ ) if $M \neq S+T$ for any proper 
submodule $T$ of $M$. A module $M$ is called hollow if every proper submodule of $M$ is small in $M$. A non-empty family $\left\{N_{\lambda}\right\}_{\lambda \in \Lambda}$ of proper submodules of a module $M$ is called coindependent if for every $\lambda \in \Lambda$ and any finite subset $F \subseteq \Lambda \backslash\{\lambda\}, N_{\lambda}+\cap_{i \in F} N_{i}=M$. A module $M$ is called to have finite hollow dimension if $\mathrm{M}$ does not contain an infinite coindependent family of submodules. Let $N$ and $L$ be submoduls of $M, N$ is called a supplement of $L$ if it is minimal with the property $M=N+L$, equivalently $M=N+L$ and $N \cap L \ll N . M$ is amply supplemented if for any two submodules $A$ and $B$ of $M$ with $M=A+B$ there exist a supplement $\mathrm{P}$ of A such that $P \leq B$. For $A \subseteq B \subseteq M, \mathrm{~A}$ is called a coessential submodule of $\mathrm{B}$ in $M$ (denoted by $\left.A \leq{ }^{c e} B\right)$ if $B / A \ll M / A$. A submodul $A$ of $M$ is called coclosed in $M$ (denoted by $A \leq^{c c} M$ ) if $A$ has no proper coessential submodule.

$M$ is called lifting, if for any submodule $N$ of $M$, there exist a direct summand $K$ of $M$ such that $K \leq N$ and $N / K \ll M / K$, equivalently $M$ is lifting if and only if $M$ is amply supplemented and every supplement submodule of $M$ is a direct summand of $M$. We say $N$ lies above $K$ in $M$ if $N / K \ll M / K$. An $R$-module $M$ is called hollow - lifting if $M$ is amply supplemented and every hollow submodule of $M$ lies above a direct summand of $M$. An internal direct sum $\bigoplus_{I} A_{i}$ of submodule of a module $M$ is called a local direct summand of $M$ if, given any finite subset $F$ of the index set $I$, the direct sum $\bigoplus_{i \in F} A_{i}$ is a direct summand of $M$. The family $\left\{M_{i} \mid i \in I\right\}$, is called relatively projective if $M_{i}$ is $M_{j}$ - projective for each $i \neq j ; i, j \in I$.

A module $M$ is called to has $C_{3}$, provided for any two direct summands $M_{1}$ and $M_{2}$ of $M$ if $M_{1} \cap M_{2}=0$, then $M_{1} \oplus M_{2}$ is a direct summand of $M$. Finally we recall that a ring $R$ is a right $V$-ring if and only if every simple module is injective, if and only if $\operatorname{Rad}(M)=0$ for all $R$-modules.

Definition 2.1 Let $N \leq M, N$ is called coessentialy finitely generated if it is contained coessentialy in a finitely generated submodule of $M$ (i.e. there exists a finitely generated submodule $H$ of $M$ such that, $N \leq^{c e} H$ in $M$ ).

Definition 2.2 A module $M$ is called finitely lifting, or f-lifting for short, if every finitely generated submodule of $M$ lies above a direct summand.

Definition 2.3 An R-module $M$ is called cf-lifting if every submodule of $M$ which contained coessentialy in a finitely generated submodule lies a bove a direct summand.

It is clear that every lifting module is cf-lifting.

Definition 2.4 $M$ is called amply supplemented with respect to coessentialy finitely generated submodules (or c.f.g. amply supplemented for short) in case for each coessentialy finitely generated submodules $X$ and $Y$ such that $M=$ $X+Y, Y$ contains a supplement of $X$. 
Definition 2.5 Let $M_{1}$ and $M_{2}$ be modules. The module $M_{1}$ is smally $M_{2}$ projective if every homomorphism $f: M_{1} \rightarrow M_{2} / A$ where $A$ is a submodule of $M_{2}$ and $\operatorname{Imf} \ll M_{2} / A$, can be lifted to a homomorphism $\rho: M_{1} \rightarrow M_{2}$.

\section{Main Results}

Lemma 3.1 If $M$ is c.f.g. amply supplemented then every submodule of $M$ which contained coessentialy in a finitely generated submodule of $M$ that is not small in $M$ lies above a supplement in $M$.

Proof. It is clear by [6, Proposition (2.2)].

Theorem 3.2 Let $M$ be an $R$-module. Then the following statements are equivalent:

1) $M$ is cf-lifting.

2) For every coessentialy finitely generated submodule $N$ of $M$, there is a decomposition $M=M_{1} \oplus M_{2}$ such that $M_{1} \subseteq N, N \cap M_{2} \ll M$.

3) Every coessentialy finitely generated submodule $N$ of $M$ can be written as $N=N_{1} \oplus N_{2}$ with $N_{1}$ is direct summand of $M$ and $N_{2} \ll M$.

4) $M$ is c.f.g amply supplemented and every coessentialy finitely generated coclosed submodule of $M$ is a direct summand of $M$.

Proof. It is clear By [6, Proposition (1.1)].

Theorem 3.3 If $M$ is cf-lifting then $M$ is f-lifting.

Proof. Let $A$ be a finitely generated submodule of $M$ then $A \leq^{c e} A$ in $M$ hence there exists a summand $K$ of $M$ such that $K \leq{ }^{c e} A$ in $M$ then $M$ is f-lifting.

Lemma 3.4 Let $M$ be an $R$-module and $K \subseteq L$ be submodules of $M$. Then if $K \leq^{c c} M$, then $K \leq^{c c} L$ and the converse is true if $L \leq{ }^{c c} M$.

Proof. By [3, Lemma 2.6]

Theorem 3.5 Any coclosed submodule (and hence any direct summand) of a cf-lifting module is cf-lifting.

Proof. Let $K$ be a coclosed submodule of $M$ and $M$ be a cf-lifting module. Let $N \leq{ }^{c c} K$ and $N \leq{ }^{c e} H$ in $K$ such that $H$ is a finitely generated submodule of $K$. Now $H / N \ll K / N \leq M / N$ implies that $H / N \ll M / N$ i.e. $N \leq{ }^{c e} H$ in $M$. Since $N \leq{ }^{c c} K$ and $K \leq{ }^{c c} M$, then $N \leq{ }^{c c} M$. So $N$ is a direct summand of $M$ and hence a direct summand of $K$. It means $K$ is a cf-lifting modules. 
Theorem 3.6 For noetherian $R$-module $M$, the following statements are equivalent:

1) $M$ is cf-lifting.

2) $M$ is f-lifting.

3) $M$ is lifting.

Proof. $1 \Rightarrow 2$ By the above Proposition.

$2 \Rightarrow 3$ Since $M$ is noetherian, every submodule of $M$ is finitely generated so $M$ is lifting.

$3 \Rightarrow 1$ It is trivial.

Lemma 3.7 Let $M$ be an $R$-module with finite hollow dimension. Then $M$ is hollow-lifting if and only if $M$ is lifting.

Proof. By [6, Corollary (1.5)].

Lemma 3.8 Let $M$ be an hollow-lifting module and $K \leq^{c c} M$ with finite hollow dimension. Then $K$ is a direct summand of $M$.

Proof. By [6, Lemma (1.4)].

Theorem 3.9 Let $M$ be an $R$-module with finite hollow dimension. If $M$ is hollow-lifting module then $M$ is cf-lifting.

Proof. It follows immediately from Lemma 1.11 and Lemma 1.12.

Lemma 3.10 Let $M=M_{1} \oplus \ldots \oplus M_{n}$ be a finite direct sum of relatively projective modules $M_{i}$. Then $M$ is lifting if and only if $M$ is amply supplemented and $M_{i}$ is lifting for all $1 \leq i \leq n$.

Proof. By [4, Corollary (2.9)].

Lemma 3.11 Let $M=M_{1} \oplus M_{2}$ be a weakly supplemented module. Suppose that for every coclosed submodule $N$ of $M$ such that $M=N+M_{1}$ or $M=$ $N+M_{2}, N$ is a direct summand of $M$. Let $K$ be a coclosed submodule of $M$ such that every submodule of $M / K$ has an s-closure in $M / K$. Then $K$ is a direct summand of $M$.

Proof. By [4, Proposition (1.8)].

Theorem 3.12 Let $M=M_{1} \oplus M_{2}$ be an amply supplemented module. Suppose that every coclosed submodule $K$ of $M$ with $M=K+M_{1}$ is a direct summand of $M$ or every coclosed submodule $K$ of $M$ which is coessentialy finitely generated such that $M=K+M_{2}$, is a direct summand of $M$. Then $M$ is cf-lifting. 
Proof. It follows from Lemma 2.1 and Lemma 2.2.

Theorem 3.13 Let $M$ be an amply supplemented module and $M=M_{1} \oplus$ $M_{2}$ is a direct sum of relatively projective modules $M_{1}$ and $M_{2}$ such that $M_{1}$ is lifting and $M_{2}$ is cf-lifting. Then $M$ is cf-lifting.

Proof. Let $N$ be a coclosed submodule of $M$ such that $M=N+M_{1}$. There exists a submodule $N^{\prime}$ of $N$ such that $M=N^{\prime}+M_{1}$. Since $M / N^{\prime}$ is lifting and $N / N^{\prime}$ is coclosed in $M / N^{\prime}, N / N^{\prime}$ is direct summand of $M / N^{\prime}$. Therefore $N$ is direct summand of $M$. Hence it follows from Proposition $2.3 M$ is cf-lifting. For the case $M=N+M_{2}$ it is clear by Proposition 2.3.

Theorem 3.14 Let $M$ be a module such that every submodule of $M$ has a coclosure in $M$ and $M$ has $C_{3}$. If every local direct summand of $M$ is a direct summand, then $M$ is cf-lifting if and only if it is lifting.

Let $0 \neq K \leq \leq^{c c} M$. For $0 \neq x \in K, x R$ has coclosure in $M$ such as $A$. $A$ is coclosed in $M$ and $A$ is coessential in $x R$, so $A$ is a direct summand of $M$ $(A \subseteq x R \subset K \subseteq M)$. By Zorn's Lemma there exists a maximal local direct summand $N=\oplus_{I} A_{i}$ where each $A_{i} \subset K$. By hypothesis, $N$ is direct summand of $M$ i.e. $M=N \oplus N^{\prime}$ for some submodule $N^{\prime}$ of $M$, so $K=N \oplus\left(K \cap N^{\prime}\right)$. Assume that $K \cap N^{\prime} \neq 0$. Suppose that for $0 \neq x \in N^{\prime} \cap K, x R$ has a coclosure $B$ in $M$.Hence $B$ is a direct summand of $M$ and $B \cap N=0$ so $N \oplus B$ is a local direct summand of $M$, a contradiction with maximality of $N$. Hence $K \cap N^{\prime}=0$ so $K=N$ a direct summand of $M$.

Theorem 3.15 $A$ ring $R$ is a $V$-ring if and only if for any $R$-module $M$, every submodule of $M$ is coclosed in $M$.

Proof. By [3, Lemma 2.1].

Lemma 3.16 LetR be a $V$-ring. An $R$-module $M$ is lifting if and only if it is semisimple.

Proof. It is clear by above Proposition.

Corollary 3.17 Let $R$ be $a V$-ring and $M$ be a noetherian $R$-module then $M$ is cf-lifting if and only if it is semisimple.

Proof. By Proposition (2.6)and Lemma (2.7).

Lemma 3.18 Let $M_{1}$ and $M_{2}$ be modules and $M=M_{1} \oplus M_{2}$. The following statements are equivalent:

1) $M_{1}$ is smally $M_{2}$-projective. 
2) For every submodule $N$ of $M$ such that $\left(N+M_{1} / N\right) \ll M / N$, there exists a submodule $N^{\prime}$ of $N$ such that $M=N^{\prime} \oplus M_{2}$.

Proof. By [4, Lemma (2.4)].

Lemma 3.19 Let $M_{1}$ be any module and $M_{2}$ a lifting module and let $M=$ $M_{1} \oplus M_{2}$. If $M_{1}$ is smally $M_{2}$-projective, then every coclosed submodule $N$ of $M$ such that $\left(N+M_{1}\right) / N \ll M / N$ is a direct summand.

Proof. By [4, Lemma (2.7)].

Theorem 3.20 Let $M_{1}$ be a lifting module and $M_{2}$ be a cf-lifting modules and $M=M_{1} \oplus M_{2}$ be an amply supplemented module. If one of the following conditions holds, then $M$ is cf-lifting.

1) $M_{1}$ is smally $M_{2}$-projective and every coclosed submodule $N$ of $M$ such that $M=N+M_{1}$ is a direct summand.

2) $M_{1}$ and $M_{2}$ are relatively smally projective and every coclosed submodule $N$ of $M$ such that $M=N+M_{1}=N+M_{2}$ is a direct summand.

3) $M_{2}$ is $M_{1}$-projective and $M_{1}$ is smally $M_{2}$-projective .

4) $M_{1}$ is semisimple and smally $M_{2}$-projective.

Proof. It is clear by [4, Theorem 2.8].

\section{References}

[1] F. W. Anderson and K. R. Fuller, Rings and Categories of Modules, Spring Verlag, New York 1974.

[2] J. Clark, C. Lomp, N. Vanaja and R. Wisbauer, Lifting Modules, Frontiers in Mathematics, Birkhaeuser Basel 2006.

[3] L. Ganesan and N. Vanaja, Modules for which every Submodule has a Unique Coclouser, Comm. Algebra, 30 (2002), 2355-2377.

[4] D. Keskin, On Lifting Modules, Comm. Algebra, 28 (2000), 3427-3440.

[5] D. Keskin, On Coclosed Submodules, Indian J. pure appl. Math.,36 (2005), 135-144.

[6] C. Lomp, , On Dual Goldie Dimension, Diplomarbeit (M. Sc. Thesis),Doesseldorf (1996).

[7] S. H. Mohamed and B. J. Muller, Continuous and discrite modules, Londen Math. Soc. LNS 147 Cambridge Univ. press, Cambridge, 1990. 
[8] R. Wisbauer, Foundations of Module and Ring Theory, Gordon and Breach: Reading, 1991.

Received: March 14, 2007 